\title{
Superradiant instability of Kerr-de Sitter black holes in scalar-tensor theory
}

\author{
Cheng-Yong Zhang, Shao-Jun Zhang and Bin Wang \\ Department of Physics and Astronomy, Shanghai Jiao Tong University, \\ Shanghai 200240, China \\ E-mail: zhangcy@sjtu.edu.cn, sjzhang84@sjtu.edu.cn, wang_b@sjtu.edu.cn
}

\begin{abstract}
We investigate in detail the mechanism of superradiance to render the instability of Kerr-de Sitter black holes in scalar-tensor gravity. Our results provide more clues to examine the scalar-tensor gravity in the astrophysical black holes in the universe with cosmological constant. We also discuss the spontaneous scalarization in the de Sitter background and find that this instability can also happen in the spherical de Sitter configuration in a special style.
\end{abstract}

KEYwords: Black Holes, Classical Theories of Gravity

ArXiv EPRINT: 1405.3811 


\section{Contents}

1 Introduction 1

2 Kerr-de Sitter black hole in scalar-tensor theory 2

3 Perturbation equations and superradiance 5

3.1 Perturbation equations 5

$\begin{array}{lll}3.2 & \text { Superradiance condition } & 6\end{array}$

4 Numerical results of the superradiant instability $\quad 7$

$\begin{array}{lll}4.1 & a=0.99 & 7\end{array}$

$\begin{array}{ll}4.2 a=0.7 \text { and } a=0.3 & 10\end{array}$

4.3 The minimum $a$ to accommodate the superradiant instability 12

5 Spontaneous Scalarization $\quad 13$

$\begin{array}{llr}6 & \text { Summary and discussion } & 16\end{array}$

\section{Introduction}

Scalar-tensor (ST) theory is an alternative theory that describes gravity beyond Einstein's general relativity (GR). This theory was originally conceived by Jordan and later generalized by Brans and Dicke [1]. The primary motivation comes from cosmology to search for a theory incorporating Mach's principle which is not explicitly embodied in GR. In the ST theory, the gravitational coupling is determined by all matter in the universe. The cosmological distribution of matter will affect local gravitational experiments [2, 3]. Currently, interests in ST theory come from many aspects. First, a fundamental scalar field coupled to gravity is an unavoidable feature of unified theory such as supergravity, string theory [4], etc. Second, the inflationary scenarios of universe and dark energy may be described by ST theory $[2,3]$.

Experimentally, the differences between ST theory and GR are too small to be detected in the weak field regime, i.e, in the solar system $[5,6]$. Particular interest of the phenomenology of the ST theory is in the strong gravity regime. It is expected that the strong gravity test can be a possible way to distinguish the ST theory from GR.

For compact stars in ST theory, unexpected phenomenon has been discovered compared with GR [7-11]. For the black hole (BH) background in ST theory, when the scalar field settles to a constant, we can get the same BH solution as that in GR [12, 13]. However the perturbations will behave differently as the two theories have different dynamics $[14,15]$. It has been shown that compared with the Kerr BH background in GR, the existence of 
a scalar mode in the spectrum of perturbations around a Kerr BH in the ST theory leads to remarkable effects $[16,17]$. When the matter configuration surrounding the Kerr black hole is dense enough, the $\mathrm{BH}$ will be forced to develop scalar hair. On the other hand, when the BH rotates sufficiently fast, superradiant instability can occur. Neither of these instabilities has been observed in the Kerr BH solution background in GR. This gives the hope that BHs can be used as probes in order to distinguish ST theory from GR.

Considering that our universe has a positive cosmological constant [18], we will extend the discussions in the asymptotically flat Kerr BH backgrounds $[16,17]$ to Kerr-de Sitter configurations. We will examine in detail the mechanism that can render the instability of Kerr-de Sitter BHs driven by superradiance in the ST gravity. To trigger superrandiant instability, two necessary conditions need to be satisfied [19], namely that the BH must have superradiance and the existence of a potential well outside the $\mathrm{BH}$ to trap the scattered wave. The superradiant instability in GR has been discussed thoroughly, see review [20] and references therein. In this work, we will examine how the instability depends on the matter profile, the rotation of the $\mathrm{BH}$ and the cosmological constant. Furthermore we will investigate the spontaneous scalarization in the Kerr-de Sitter BH background. Comparing with the stability analysis in GR [21] where no instability was found for Kerr-de Sitter $\mathrm{BH}$ configurations, our results will provide more possible potential observational clues to distinguish the ST gravity from GR in the BH backgrounds in the real universe.

The organization of the paper is as follows. In section II, we will briefly present the framework of ST theory and describe the Kerr-de Sitter BH. In section III, we will derive the perturbation equation of the scalar field. In section IV, we will calculate the frequencies of the perturbation numerically and list our results. In section V, we will discuss the spontaneous scalarization for spherical de Sitter BHs. The last section is devoted to discussion and summary.

\section{Kerr-de Sitter black hole in scalar-tensor theory}

The general action of the ST theory in the Jordan frame is $[2,3]$

$$
\begin{aligned}
S= & \frac{1}{16 \pi} \int d^{4} x \sqrt{-g}\left(F(\phi) R^{J}-Z(\phi) g_{\mu \nu} \partial^{\mu} \phi \partial^{\nu} \phi-U(\phi)\right) \\
& +S_{m}\left(\Psi_{m} ; g_{\mu \nu}\right),
\end{aligned}
$$

where $R^{J}$ is the Ricci scalar of metric $g_{\mu \nu}, \phi$ is a scalar field and $U(\phi)$ is the scalar field potential. $S_{m}\left(\Psi_{m} ; g_{\mu \nu}\right)$ is the action describing matter $\Psi_{m}$ which is minimally coupled to $g_{\mu \nu}$ and $\phi$. Functions $F, Z$ and $U$ represent specific theories within the class, up to a degeneracy due to the freedom of redefining the scalar [22]. When $F=\phi, Z=\omega_{0} / \phi$ and $U=0$, one gets the Brans-Dicke theory [1]. The ST theory can be viewed as a low-energy limit of a bosonic string theory when $F=\phi, Z=-\phi^{-1}$.

Performing a conformal transformation and the field redefinition

$$
\begin{aligned}
g_{\mu \nu}^{E} & =F(\phi) g_{\mu \nu}, \\
\Phi(\phi) & =\frac{1}{\sqrt{4 \pi}} \int d \phi\left(\frac{3}{4} \frac{F^{\prime}(\phi)^{2}}{F(\phi)^{2}}+\frac{1}{2} \frac{Z(\phi)}{F(\phi)}\right)^{1 / 2},
\end{aligned}
$$




$$
\begin{aligned}
& A(\Phi)=F^{-1 / 2}(\phi), \\
& V(\Phi)=\frac{U(\phi)}{F^{2}(\phi)},
\end{aligned}
$$

we get the action in the Einstein frame (For more details, see $[2,3]$ )

$$
\begin{aligned}
S= & \frac{1}{16 \pi} \int d^{4} x \sqrt{-g^{E}}\left(R^{E}-8 \pi g_{\mu \nu}^{E} \partial^{\mu} \Phi \partial^{\nu} \Phi-V(\Phi)\right) \\
& +S_{m}\left(\Psi_{m} ; A(\Phi)^{2} g_{\mu \nu}^{E}\right) .
\end{aligned}
$$

In Einstein frame, the scalar field is minimally coupled to gravity, but the matter field is coupled to the metric $A(\Phi)^{2} g_{\mu \nu}^{E}$. The field equations in the Einstein frame can be derived by varying action $(2.3)$.

$$
\begin{aligned}
G_{\mu \nu}^{E} & =8 \pi\left(T_{\mu \nu}^{E}+\partial_{\mu} \Phi \partial_{\nu} \Phi-\frac{g_{\mu \nu}^{E}}{2}(\partial \Phi)^{2}\right)-\frac{g_{\mu \nu}^{E}}{2} V(\Phi), \\
\nabla_{\mu} \nabla^{\mu} \Phi & =-\frac{A^{\prime}(\Phi)}{A(\Phi)} T^{E}+\frac{V^{\prime}(\Phi)}{16 \pi} .
\end{aligned}
$$

Here $T_{\mu \nu}^{E} \equiv-\frac{2}{\sqrt{-g^{E}}} \frac{\delta S_{m}}{\delta g_{E}^{\mu \nu}}$. We assume that $\Phi=\Phi_{0}=$ const is a solution to the above equations and around $\Phi_{0}$ we have the following analytical expansions

$$
\begin{aligned}
& V(\Phi)=\sum_{n=0} V_{n}\left(\Phi-\Phi_{0}\right)^{n}, \\
& A(\Phi)=\sum_{n=0} A_{n}\left(\Phi-\Phi_{0}\right)^{n} .
\end{aligned}
$$

For abbreviations, we denote $\varphi \equiv \Phi-\Phi_{0}$ and $\alpha_{n} \equiv A_{n} / A_{0}$. Then to the order $\mathcal{O}(\varphi)$, the equations of motion become

$$
\begin{aligned}
G_{\mu \nu}^{E}+\frac{V_{0}}{2} g_{\mu \nu}^{E} & =8 \pi T_{\mu \nu}^{E}-\frac{g_{\mu \nu}}{2} V_{1} \varphi, \\
\nabla_{\mu} \nabla^{\mu} \varphi & =-\alpha_{1} T^{m}+\frac{V_{1}}{16 \pi}+\left(\alpha_{1}^{2}-2 \alpha_{2}\right) T^{m} \varphi+\frac{V_{2}}{8 \pi} \varphi .
\end{aligned}
$$

The term $V_{0}$ is related to the cosmological constant $\Lambda \equiv \frac{V_{0}}{2} . \quad V_{1}$ is a linear potential which will be dropped off since linear potential is seldom met in nature. $V_{2}$ is related to a standard mass term. $\alpha_{1}$ describes the effective coupling between the scalar and matter. To have a constant scalar solution $\Phi=\Phi_{0}=$ const as we mentioned above, from (2.9) we should set $\alpha_{1}=0$. In addition, observations, such as weak gravity constraints and tests for violation of the strong equivalence principle, seem to require $\alpha_{1}$ to be negligibly small [23, 24]. The configuration with constant scalar and $\alpha_{1} \simeq 0$ was argued most likely as an approximate solution in most viable ST theories [16, 17]. Finally, the equations of motion can be reduced to

$$
\begin{aligned}
G_{\mu \nu}^{E}+\Lambda g_{\mu \nu}^{E} & =8 \pi T_{\mu \nu}^{E}, \\
\nabla_{\mu} \nabla^{\mu} \varphi & =\left(\frac{V_{2}}{8 \pi}-2 \alpha_{2} T^{m}\right) \varphi .
\end{aligned}
$$




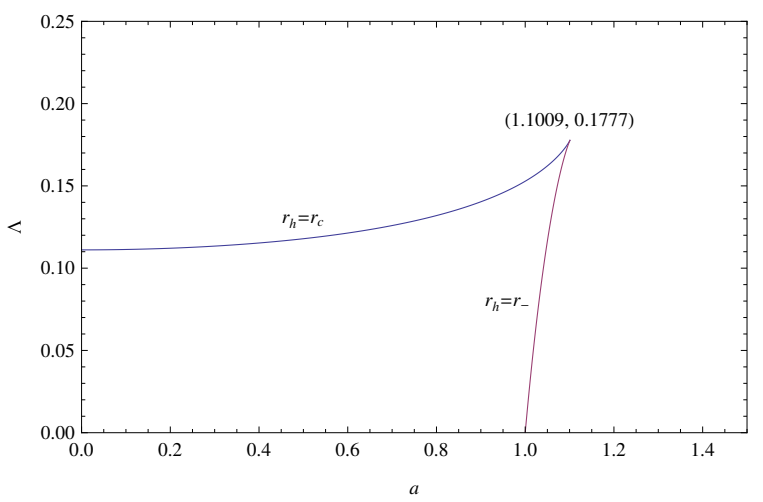

Figure 1. Parameter space for Kerr-de Sitter BH in $a-\Lambda$ plane with fixed $M=1$. Kerr-de Sitter $\mathrm{BH}$ solutions are allowed for the parameters $(a, \Lambda)$ in the region between the two curves.

Here $\mu_{s}^{2} \equiv \frac{V_{2}}{8 \pi}-2 \alpha_{2} T^{m}$ plays the role of effective mass square of the scalar field. When the backreaction of the matter field on the spacetime geometry is negligible ("the probe limit"), from (2.10) we can have the Kerr-de Sitter BH as a solution. In Boyer-Lindquist coordinates, the metric reads

$$
\begin{aligned}
d s^{2}= & -\frac{\Delta_{r}}{(1+\alpha)^{2} \rho^{2}}\left(d t-a \sin ^{2} \theta d \phi\right)^{2}+\frac{\Delta_{\theta} \sin ^{2} \theta}{(1+\alpha)^{2} \rho^{2}}\left(a d t-\left(a^{2}+r^{2}\right) d \phi\right)^{2} \\
& +\frac{\rho^{2}}{\Delta_{r}} d r^{2}+\frac{\rho^{2}}{\Delta_{\theta}} d \theta^{2}
\end{aligned}
$$

in which

$$
\begin{aligned}
\alpha & =\frac{\Lambda a^{2}}{3}, \Delta_{\theta}=1+\alpha \cos ^{2} \theta, \rho^{2}=r^{2}+a^{2} \cos ^{2} \theta, \\
\Delta_{r} & =\left(a^{2}+r^{2}\right)\left(1-\frac{\alpha}{a^{2}} r^{2}\right)-2 M r .
\end{aligned}
$$

For some ranges of parameters, $\Delta_{r}=0$ exists four real roots. Three positive real roots correspond to three horizons: the inner BH Cauchy horizon $r_{-}$, the outer BH event horizon $r_{h}$ and the cosmological horizon $r_{c}$. The remaining negative root has no physical meaning. We focus only on cases in which $\Delta_{r}=0$ has four real roots, so that we have Kerr-de Sitter BH. In figure 1, we plot the allowed parameter space. We can see that values of $(a, \Lambda)$ should lie in the region between two curves where we fix $M=1$. The upper nearly horizontal curve corresponds to the degeneracy of the cosmological horizon and the $\mathrm{BH}$ event horizon $r_{h}=r_{c}$, while the lower nearly vertical curve represents the degeneracy between the $\mathrm{BH}$ event horizon and the Cauchy horizon $r_{h}=r_{-}$. The two curves intersect at the point $(a, \Lambda) \sim(1.1009,0.1777)$, at which $r_{-}=r_{h}=r_{c}$ occurs.

The surface gravities $\kappa$ and angular velocities $\Omega$ on horizons are [21]

$$
\kappa_{i}=\frac{1}{2(1+\alpha)\left(r_{i}^{2}+a^{2}\right)} \frac{d \Delta_{r}\left(r_{i}\right)}{d r}, \Omega_{i}=\frac{a}{r_{i}^{2}+a^{2}},
$$

where $r_{i}=\left\{r_{h}, r_{c}\right\}$. 
In this paper, we will mainly focus on this Kerr-de Sitter BH configuration in the probe limit and study its superradiant stability under scalar perturbation. Different from the stability analysis in [21], we will look for separable solutions of the Klein-Gordon equations with effective mass $\mu_{s}$, not just the canonical mass of a massive scalar field. The effective mass reflects the influence of the ST theory in the presence of matter surrounding the $\mathrm{BH}$.

\section{Perturbation equations and superradiance}

\subsection{Perturbation equations}

The dynamics of the scalar field in Kerr-de Sitter background is described by (2.11). To make comparison with the result of Kerr $\mathrm{BH}$ in the asymptotically flat spacetime reported in $[16,17]$, we take the following form for the effective mass

$$
\mu_{s}^{2}(r)=\frac{2 \Lambda}{3}+\frac{G(r)}{\rho^{2}}, G(r)=\beta \Theta\left(r-r_{0}\right)\left(r-r_{0}\right)\left(\frac{1}{r^{n}}-\frac{1}{r_{c}^{n}}\right),
$$

which can reduce to the form in Kerr case $[16,17]$ when the cosmological constant $\Lambda \rightarrow 0$. Here $\Theta\left(r-r_{0}\right)$ is a heaviside function, $r_{0}$ is the place where the matter distribution starts from and $\beta$ indicates the strength of coupling between scalar and matter field. Note that $\beta \propto \alpha_{2}$ in (2.11) and large positive values of $\alpha_{2}$ are not constrained by observations, so we can take arbitrary large $\beta$. In fact, we will find that only large enough $\beta$ can trigger the instability in the next section. The term $\frac{2 \Lambda}{3}$ plays the role of the canonical mass term of a massive scalar. For Kerr-de Sitter background, it equals to $\frac{1}{6} R_{g}$ where $R_{g}$ is the Ricci scalar of spacetime. So eq. (2.11) is equivalent to the equation of motion of a massive conformally coupled scalar.

The Klein-Gordon equation (2.11) is separable when effective mass (3.1) is adopted. By assuming a separation $\varphi=R(r) S(\theta) e^{-i \omega t+i m \phi}$, the angular part of (2.11) turns out to be

$$
\frac{\partial_{\theta}\left(\sin \theta \Delta_{\theta} \partial_{\theta} S\right)}{\sin \theta S}-(1+\alpha)^{2} \frac{B^{2}}{\Delta_{\theta}}-2 \alpha \cos ^{2} \theta=-\lambda,
$$

with $\lambda$ the separation constant and $B \equiv a \omega \sin \theta-\frac{m}{\sin \theta}$. For Schwarzschild BH, $a=\alpha=0$, the separation constant $\lambda=l(l-1)$ in which $l$ is the angular momentum number. For more general BHs, there is no explicit analytical expression of $\lambda$. For Kerr spacetime, $\Delta_{\theta}=1$ and the above equation becomes a spheroidal equation which has been studied in detail in [25-32]. And $\lambda$ can be expanded as a series of $a \omega$ for small $a \omega$, whose explicit form can be found in [26, 27, 32]. For Kerr-de Sitter BH, eq. (3.2) is a generalized spheroidal equation and the explicit expansion of separation constant $\lambda$ in small $a \omega$ is [33, 34]

$$
\begin{aligned}
\lambda= & l(l+1)+\alpha\left(-l(l+1)+2 m^{2}-l^{2} H(l)+(l+1)^{2} H(l+1)\right) \\
& +[-2 m-2 m \alpha(1-H(l)+H(l+1))] a \omega \\
& +\left[H(l+1)-H(l)+\alpha\left(H(l+1)-H(l)+2\left((l+1)^{2} H(l+1)-l^{2} H(l)\right)\right.\right. \\
& \left.\left.-l H^{2}(l)+(l+1) H^{2}(l+1)-\frac{H(l) H(l+1)}{l(l+1)}\right)\right](a \omega)^{2}+\mathcal{O}\left((a \omega)^{3}\right),
\end{aligned}
$$


with $H(l) \equiv \frac{2\left(l^{2}-m^{2}\right) l}{(2 l-1)(2 l+1)}$ and $l$ being the angular momentum which takes an integer or half integer number satisfying $l \geq m$.

The radial part of $(2.11)$ is

$$
\Delta_{r} \frac{d}{d r}\left(\Delta_{r} \frac{d}{d r} R\right)+\left[(1+\alpha)^{2} K^{2}-\Delta_{r}\left(\frac{2 \alpha}{a^{2}} r^{2}+G(r)+\lambda\right)\right] R=0
$$

where $K=\omega\left(a^{2}+r^{2}\right)-a m$.

We look for the complex frequencies of the perturbation $\omega=\omega_{R}+i \omega_{I}$. We will concentrate on looking for unstable modes with $\omega_{I}>0$, which signal the instability with the growing behavior of the perturbations. To calculate the perturbation frequencies, we need to impose suitable boundary conditions,

$$
R \rightarrow \begin{cases}\left(r-r_{h}\right)^{-i\left(\omega-m \Omega_{h}\right) / 2 \kappa_{h}} & r \rightarrow r_{h}, \\ \left(r-r_{c}\right)^{i\left(\omega-m \Omega_{c}\right) / 2 \kappa_{c}} & r \rightarrow r_{c} .\end{cases}
$$

This boundary condition is physically acceptable, which corresponds to a purely ingoing wave at the $\mathrm{BH}$ event horizon and an outgoing wave at the cosmological horizon. In asymptotically flat spacetime, for example the Kerr BH, there is no cosmological horizon and the outgoing wave condition is put at the infinity. It was argued in $[35,36]$ that when the conditions at infinity in asymptotically flat BH are altered in de Sitter black hole, the usual power-law scenario in the late time tail of the perturbation in asymptotically flat $\mathrm{BH}$ does not necessarily survive in the de Sitter background. Thus it is of interest to examine the superradiant stability in the Kerr-de Sitter configuration and compare with the result disclosed in the Kerr BH [16, 17].

In general, it is difficult to solve the perturbation equations analytically. So special numerical techniques have been developed to calculate the perturbations of BHs [37, 38], such as Pöschl-Teller potential method [39-41], finite difference method [42-44], WKB approximation [45-47], continued fraction method [28-30], direct integration method (also called shooting method) $[48,49]$, etc. In this paper, we will use the direct integration method to calculate the frequencies of the perturbations. The algorithm of this method can be described briefly as follows: with the boundary conditions (3.5), we can perform two integrations: (1) solve the radial equation from the $\mathrm{BH}$ event horizon by integration to a matching point $r=r_{m}$; (2) solve the radial equation from cosmological horizon to the matching point. Then by matching the two integration results, we can derive the frequencies of the perturbations. The numerical results are given in the next section.

\subsection{Superradiance condition}

Before doing numerical calculations, let us briefly derive the superradiant condition for Kerr-de Sitter BH. Taking the following variables

$$
d r_{*}=\frac{r^{2}+a^{2}}{\Delta_{r}} d r, \Psi=\sqrt{r^{2}+a^{2}} R,
$$

the radial equation (3.4) can be written in the form of Schrödinger equation

$$
\frac{d^{2} \Psi}{d r_{*}^{2}}+\left(\omega^{2}-V\right) \Psi=0
$$


in which the effective potential reads

$$
\begin{aligned}
V & =\omega^{2}-\frac{V_{0}}{\left(r^{2}+a^{2}\right)^{2}}+\frac{\Delta_{r}}{\left(r^{2}+a^{2}\right)^{3}}\left(r \frac{d \Delta_{r}}{d r}+\frac{a^{2}-2 r^{2}}{r^{2}+a^{2}} \Delta_{r}\right), \\
V_{0} & =(1+\alpha)^{2} K^{2}-\Delta_{r}\left(\frac{2 \alpha}{a^{2}} r^{2}+G(r)+\lambda\right) .
\end{aligned}
$$

In a scattering experiment, solution of (3.7) has the following asymptotic behavior

$$
\Psi \sim \begin{cases}\mathcal{T} e^{-i(1+\alpha)\left(\omega-m \Omega_{h}\right) r_{*}} & \text { as } \quad r_{*} \rightarrow-\infty\left(r \rightarrow r_{h}\right), \\ e^{-i(1+\alpha)\left(\omega-m \Omega_{c}\right) r_{*}}+\mathcal{R} e^{i(1+\alpha)\left(\omega-m \Omega_{c}\right) r_{*}} & \text { as } \quad r_{*} \rightarrow \infty\left(r \rightarrow r_{c}\right) .\end{cases}
$$

The above boundary conditions correspond to an incident wave of unit amplitude, $e^{-i(1+\alpha)\left(\omega-m \Omega_{c}\right)}$, plunges in from the cosmological horizon and gives rise to a reflected wave of amplitude $\mathcal{R}$ going back to the cosmological horizon and a transmitted wave of amplitude $\mathcal{T}$ at the $\mathrm{BH}$ event horizon. Considering that the effective potential is real, so $\Psi^{*}$ (complex conjugate of $\Psi$ ) is also a solution of (3.7). The Wronskian of the two linearly-independent solutions, $W\left(\Psi, \Psi^{*}\right) \equiv \Psi \frac{d}{d r_{*}} \Psi^{*}-\Psi^{*} \frac{d}{d r_{*}} \Psi$, is a constant independent of $r_{*}$. Equating values of the Wronskian at the $\mathrm{BH}$ event horizon and at the cosmological horizon, we get

$$
1-|\mathcal{R}|^{2}=\frac{\omega-m \Omega_{h}}{\omega-m \Omega_{c}}|\mathcal{T}|^{2} .
$$

Then we can see that if $\left(\omega-m \Omega_{h}\right)\left(\omega-m \Omega_{c}\right)<0$, we have $|\mathcal{R}|^{2}>1$. This means that the amplitude of the reflected wave is larger than that of the incident wave, and superradiance phenomenon occurs. So we get the condition to trigger the superradiance $[50,51]$

$$
m \Omega_{c}<\omega<m \Omega_{h} .
$$

When there is no cosmological constant, from (24) and (25), we see that the superradiant condition goes back to that for the Kerr BH.

\section{Numerical results of the superradiant instability}

We list our numerical results in this section. We fix the $\mathrm{BH}$ mass parameter $M=1, n=3$ in the matter profile and the angular indexes $l=m=1$. There are four free parameters left to affect the numerical results, namely the cosmological constant $\Lambda$, the angular momentum per unit mass $a$, the location of the matter shell $r_{0}$ and the coupling between matter and the scalar field $\beta$. To see the effect of rotation on the instability, in the following we will consider three cases with $a=0.99,0.7,0.3$, respectively. And in each case, we will choose some values of $\Lambda$ to see the influence of the cosmological constant on the instability.

\section{$4.1 \quad a=0.99$}

In this subsection, we fix the angular momentum per unit mass of the $\mathrm{BH}$ and examine the influences of the other parameters on the superradiant stability. 

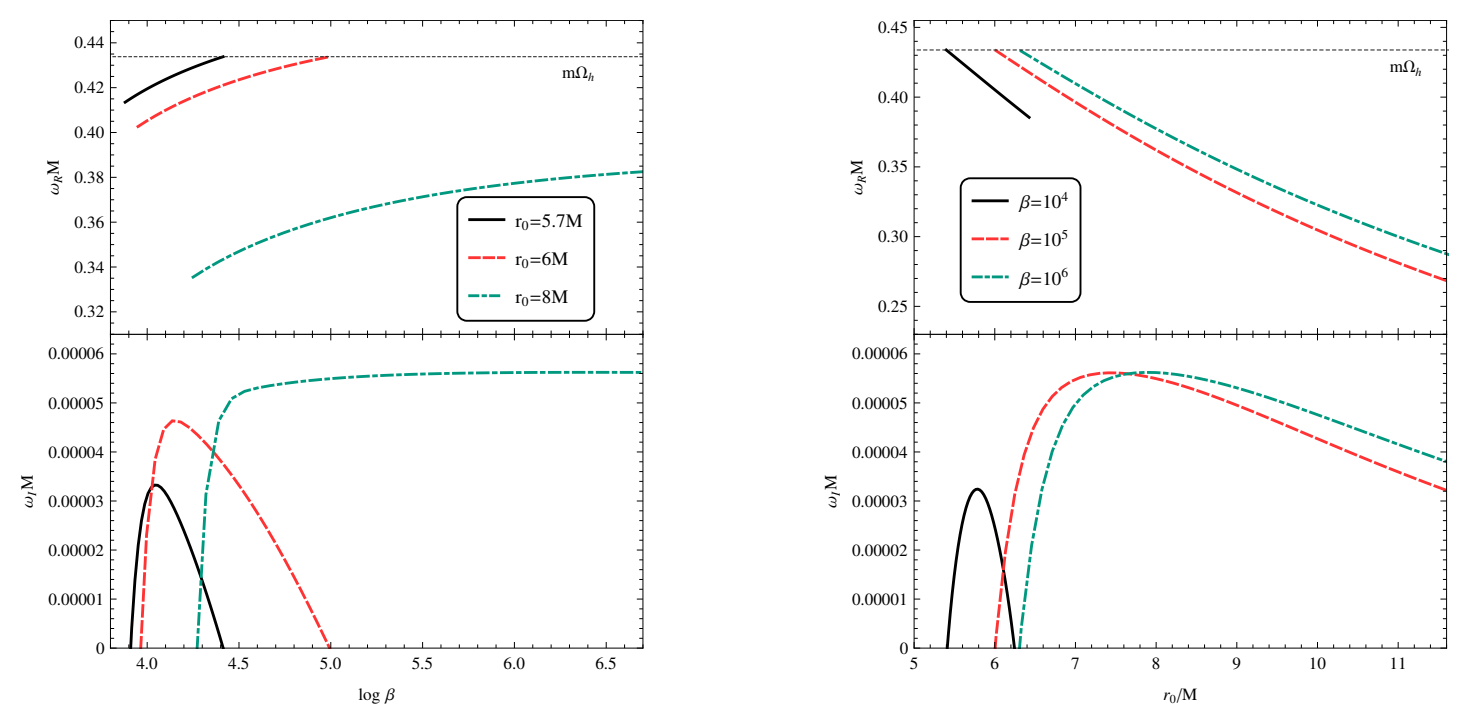

Figure 2. Superradiant instability details for Kerr BH. The outer BH event horizon $r_{h}=1.14107$ and the angular velocity on the outer horizon $\Omega_{h}=0.4338$.

In figure 2, we repeat the result of the superradiant instability in the Kerr BH background and we find that our numerical results are in good agreement with that reported in $[16,17]$. This gives us confidence in generalizing our numerical calculation to examine the stability in the Kerr-de Sitter backgrounds.

From figures 3-5, we list the superradiant instability details for the Kerr-de Sitter black hole backgrounds with the cosmological constant $\Lambda=0.0003,0.03,0.1$ respectively. The results are similar to that in the Kerr black hole case. When the real parts of the perturbation frequencies satisfy the superradiant condition (3.11), the imaginary parts of the frequencies have positive values, indicating the existence of the superradiant instability. In the figures, since $\Omega_{c}$ is too small at the cosmological horizon, it is not shown.

In the Kerr-de Sitter BH backgrounds, we see that with the increase of the cosmological constant, the $\mathrm{BH}$ event horizon $r_{h}$ increases, which leads the angular velocity on the $\mathrm{BH}$ event horizon to decrease for the $\mathrm{BH}$ with the same $a[21,52-54]$. The decrease of the upper bound in the superradiant condition (25) results in the decrease of the real part of the perturbation frequencies. This can be seen clearly from figures $3-5$. In addition, we can read from figures $3-5$ that the imaginary part of the frequencies also decreases with the increase of the cosmological constant in the Kerr-de Sitter background. Thus the superradiant instability is quenched with the increase of the cosmological constant. Physically this can be understood by looking at the effective potential in figure 6. Fixing parameters $a, r_{0}, \beta$, we see that with the increase of the cosmological constant in the Kerrde Sitter BH background, the potential well becomes shallower and the barrier to reflect radiation back becomes lower. This leads to fewer outgoing waves to be reflected back and stored in the potential well, which explains why the superradiant instability is quenched for large cosmological constant.

Comparing figures $3-5$, we find that to compensate the influence brought by increasing the cosmological constant, we need larger $\beta$ or smaller $r_{0}$ to trigger the superradiant in- 

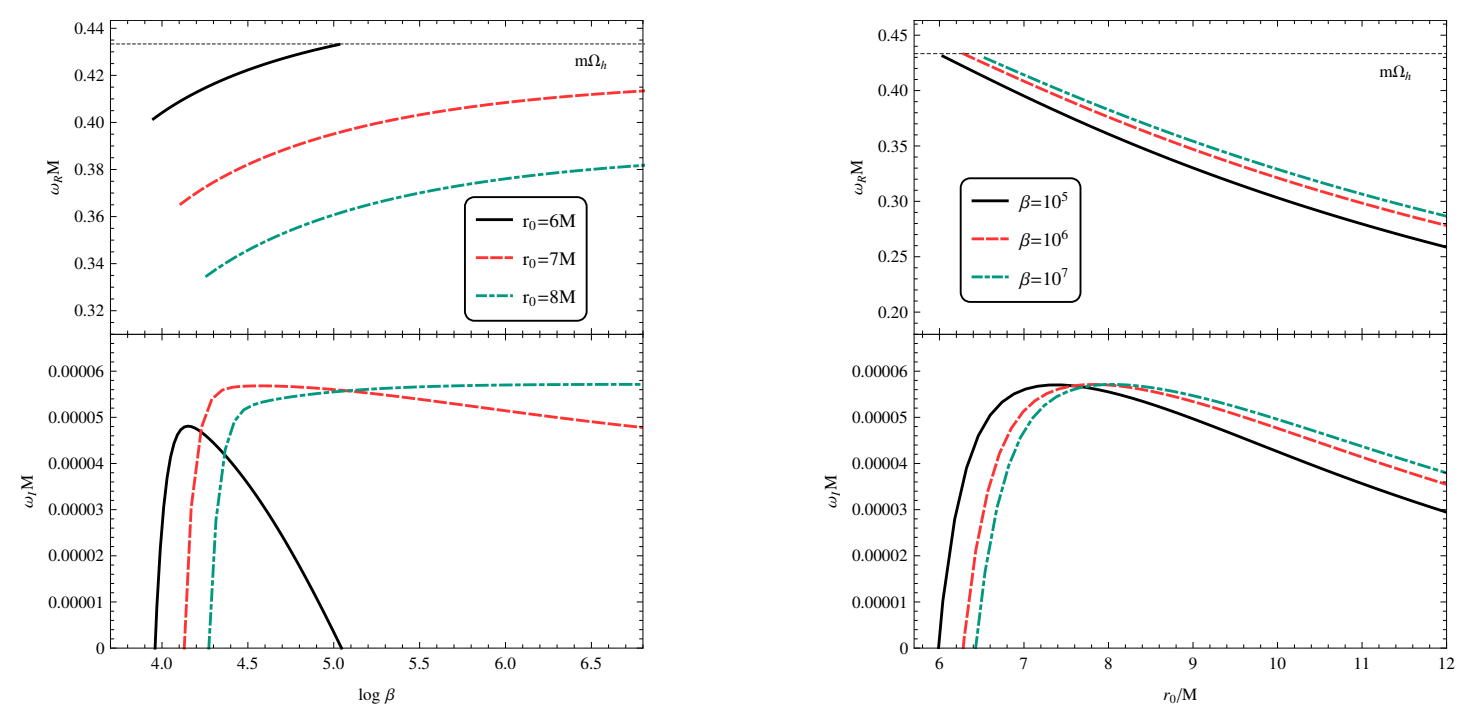

Figure 3. Superradiant instability details for Kerr-de Sitter BH with $\Lambda=0.0003$. The BH event horizon and cosmological horizon locate at $r_{h}=1.1421, r_{c}=98.9447$. The angular velocity on the BH horizon $\Omega_{h}=0.4333$.
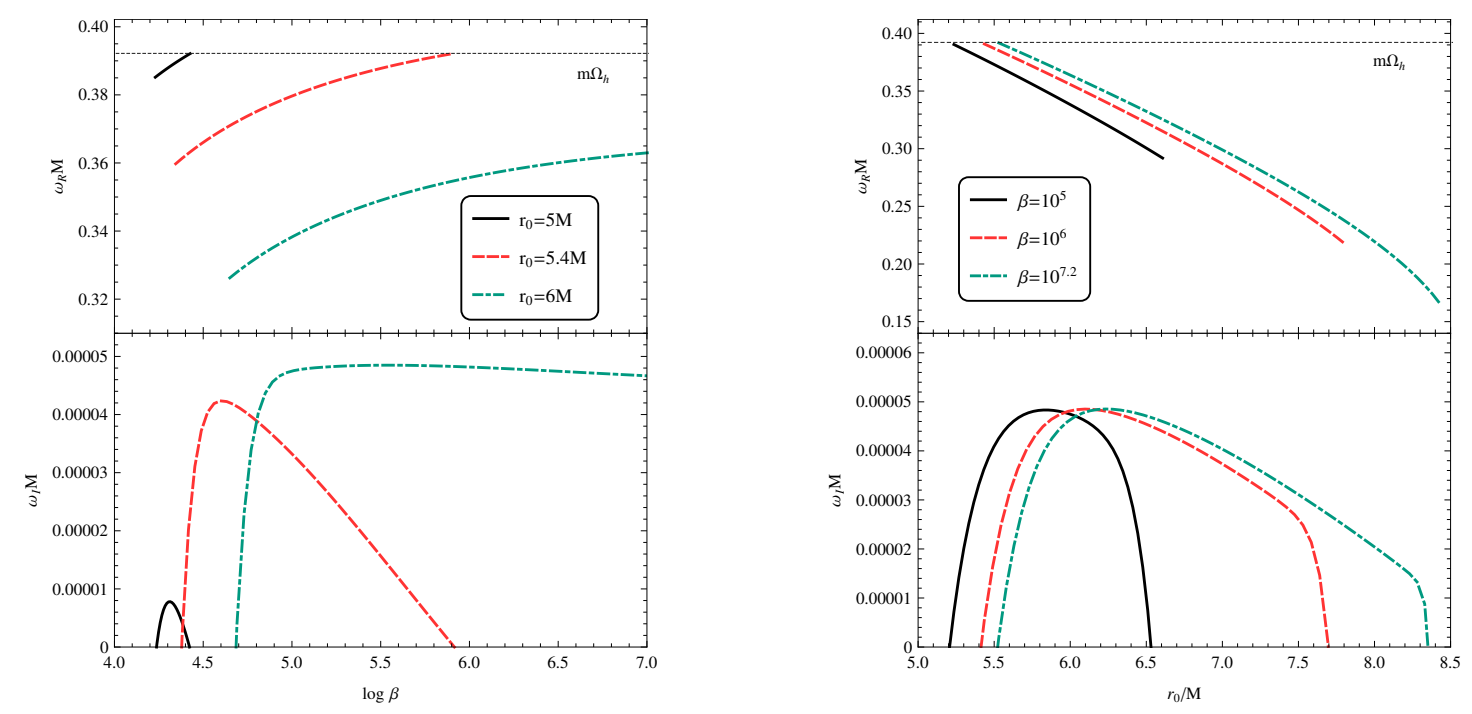

Figure 4. Superradiant instability details for Kerr-de Sitter BH with $\Lambda=0.03$. The BH event horizon and cosmological horizon locate at $r_{h}=1.2426, r_{c}=8.8077$. The angular velocity on the BH horizon $\Omega_{h}=0.3922$.

stability in the Kerr-de Sitter background. From (3.1), we can see that larger $\beta$ or smaller $r_{0}$ leads to bigger effective mass and so that higher effective potential barrier. Objective pictures of the effective potentials due to the influences of $\beta$ and $r_{0}$ are shown in figure 7 . It is clear that for fixed $r_{0}$, the increase of $\beta$ increases the potential barrier height which is able to reflect more radiations back. Fixing $\beta$, the decrease of $r_{0}$ has the same effect. Besides if we look closely at the potentials in figure 7 near the $\mathrm{BH}$ event horizon, the first potential barrier is higher so that the developed potential well is deeper for bigger $\beta$ as well 

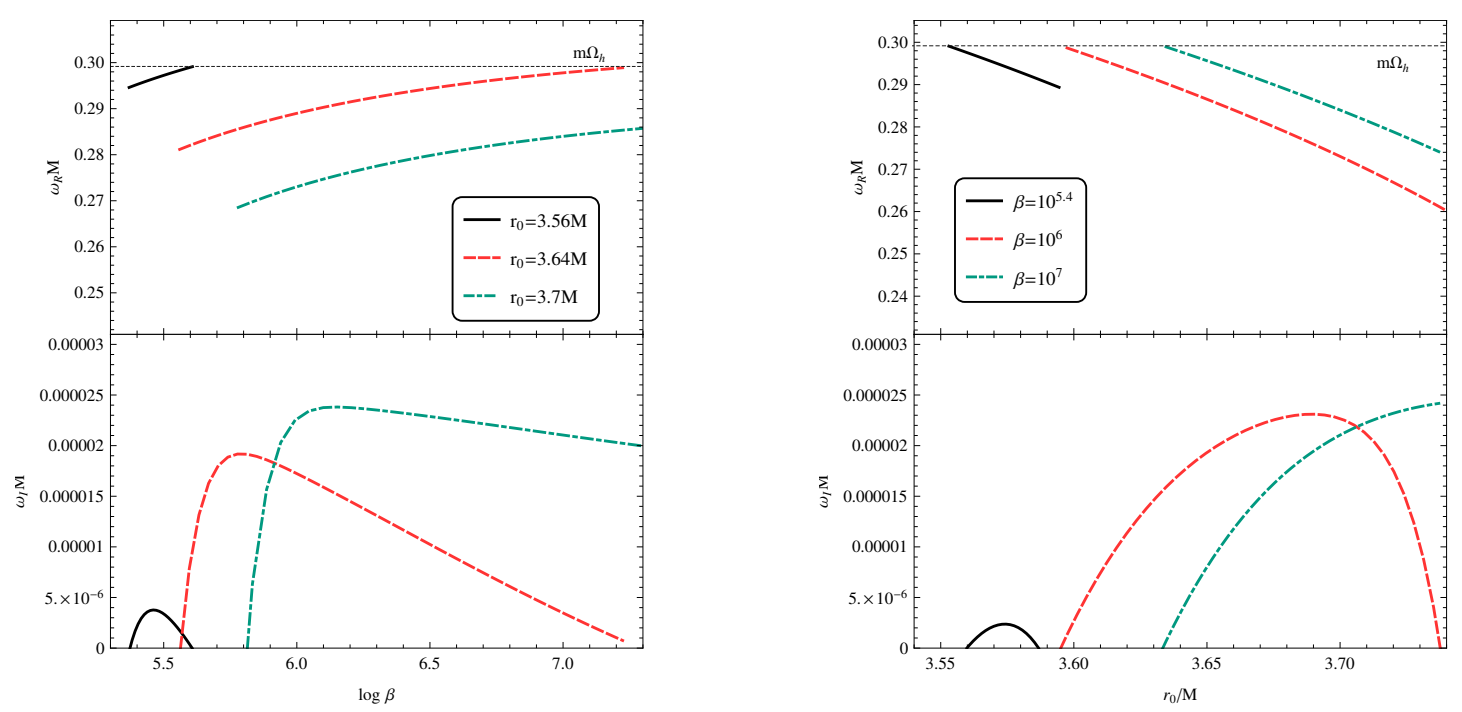

Figure 5. Superradiant instability details for Kerr-de Sitter BH with $\Lambda=0.1$. The BH event horizon and cosmological horizon locate at $r_{h}=1.5261, r_{c}=3.9722$. The angular velocity on the $\mathrm{BH}$ horizon $\Omega_{h}=0.2992$.

as for smaller $r_{0}$. Thus more outgoing wave can be reflected back by the higher potential wall and accumulated in the deeper potential well. This accounts for the phenomenon that with the increase of the cosmological constant we need to count on bigger $\beta$ and smaller $r_{0}$ to spark off the superradiant instability.

From figures 3-5 we also observe some similar properties to those of the Kerr BH reported in $[16,17]$. When the matter shell is too close to the Kerr-de Sitter BH with very small $r_{0}$, there is no superradiant instability, since the real part of the frequencies scales as $1 / r_{0}$ and too small $r_{0}$ will make the frequency violate the superradiant condition. If $r_{0}$ is not small enough, the superradiant instability can appear, but this instability will be quenched as soon as the superradiant condition is saturated. The value of $r_{0}$ where the instability starts to appear decreases with the increase of the cosmological constant adopted. When $r_{0}$ is big, the positive imaginary part of the frequencies can last for sufficiently large $\beta$. This is because that once $\beta$ is big enough to provide high enough potential barrier to bounce back the outgoing wave, further increasing $\beta$ to raise the height of the potential wall will not contribute more to the instability. We find that the above mentioned properties keep for all values of the cosmological constant in the Kerr-de Sitter BH background.

\section{$4.2 \quad a=0.7$ and $a=0.3$}

In this subsection we will fix $\Lambda=0.03$ and show the results of superradiant instability for $a=0.7$ and $a=0.3$. Combining figures 8,9 and figure 4 , we can show the influence of the angular momentum per unit mass on the superradiant instability for the Kerr-de Sitter BH background.

From figures 8,9 and combining with figure 4 for $a=0.99$, we can see that when $a$ decreases, the upper limit for the superradiant condition decreases which forces the upper value of the real part of the frequency to decrease. Considering that the real part of 


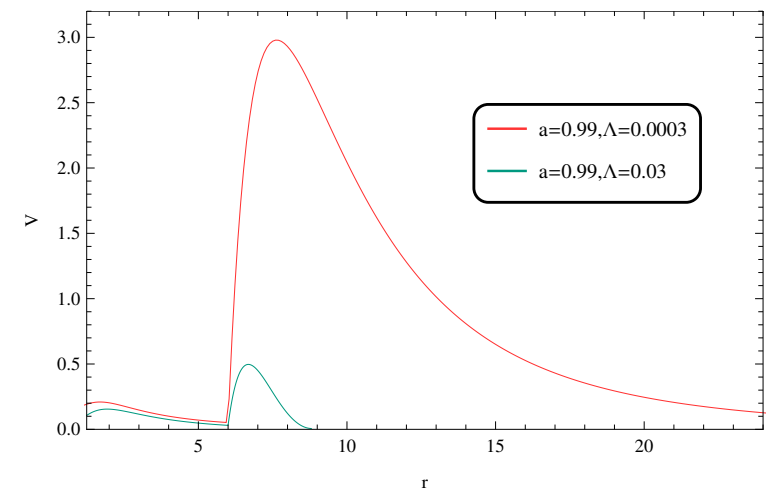

Figure 6. Effective potential behavior for the change of the cosmological constant. We fixed $a=0.09, \beta=10^{4.81}, r_{0}=6 M$.
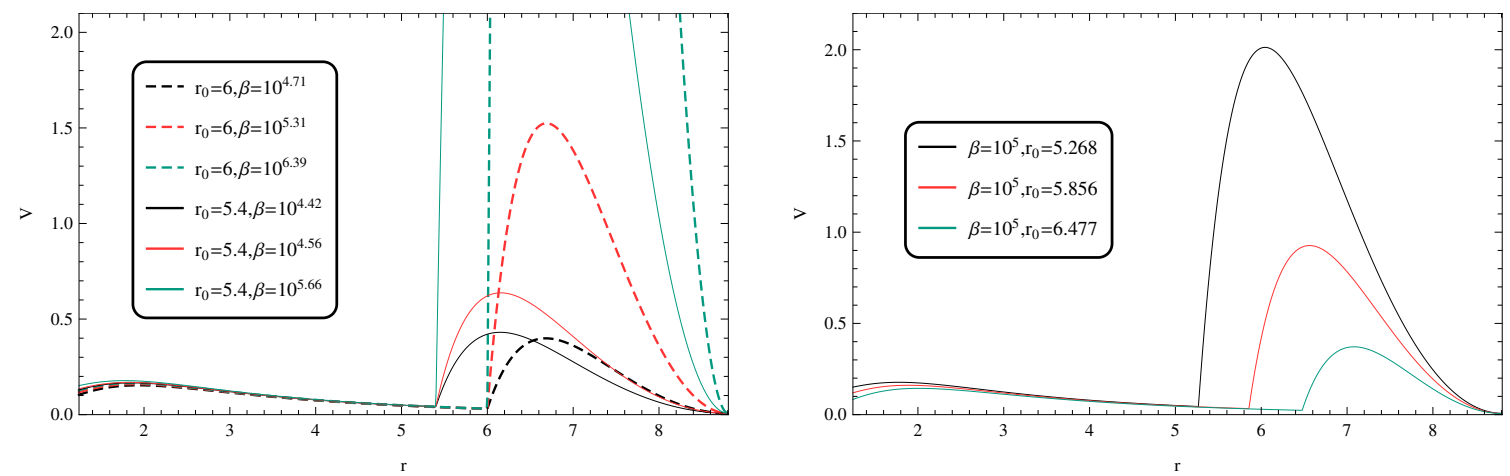

Figure 7. (Left) Effective potential behavior for the change of $\beta$. We fixed $a=0.09, \Lambda=0.03$. The solid lines are for $r_{0}=5.4$ and dashed lines are for $r_{0}=6$. (Right) Effective potential behavior for the change of $r_{0}$. We fixed $a=0.99, \Lambda=0.03, \beta=10^{5}$.
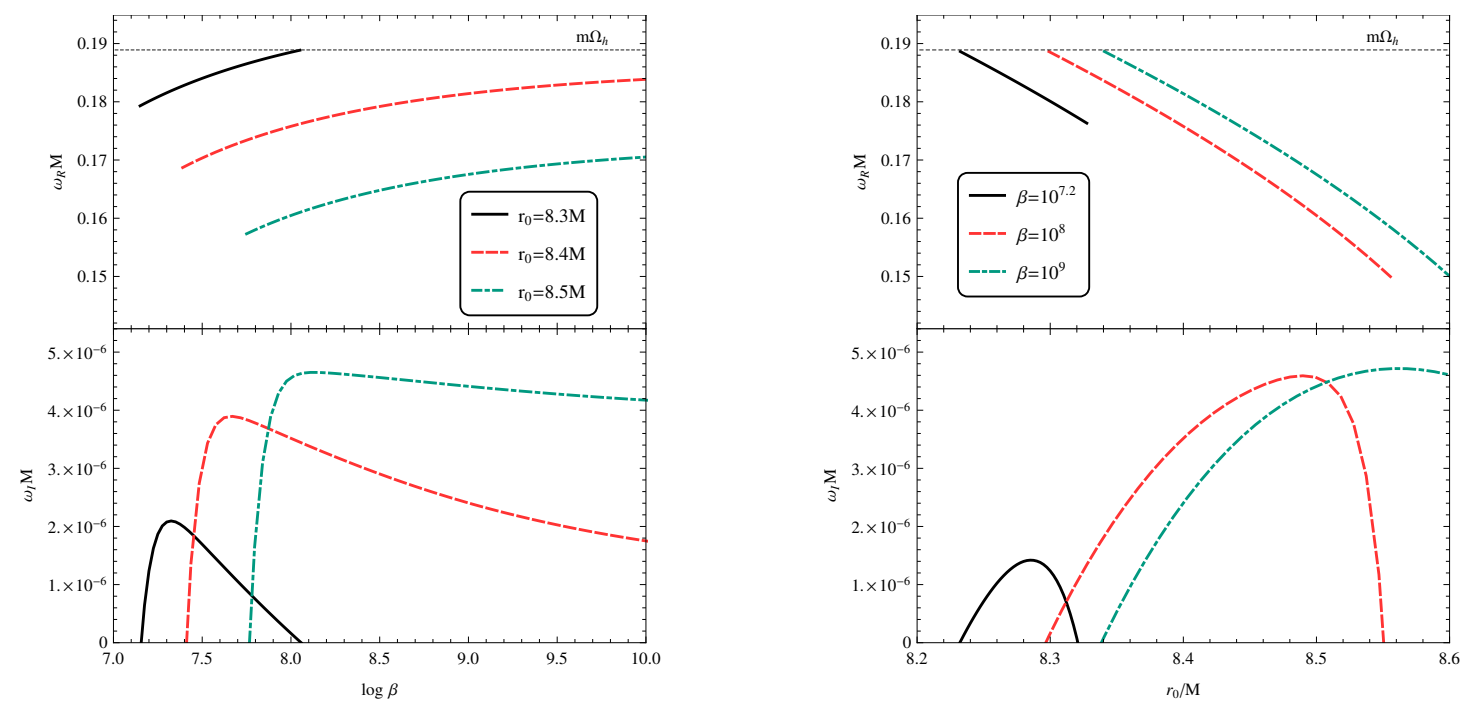

Figure 8. Superradiant instability details for Kerr-de Sitter BH with $a=0.7, \Lambda=0.03$. The BH event horizon and cosmological horizon locate at $r_{h}=1.7932, r_{c}=8.7984$. The angular velocity on the black hole horizon $\Omega_{h}=0.1889$. 

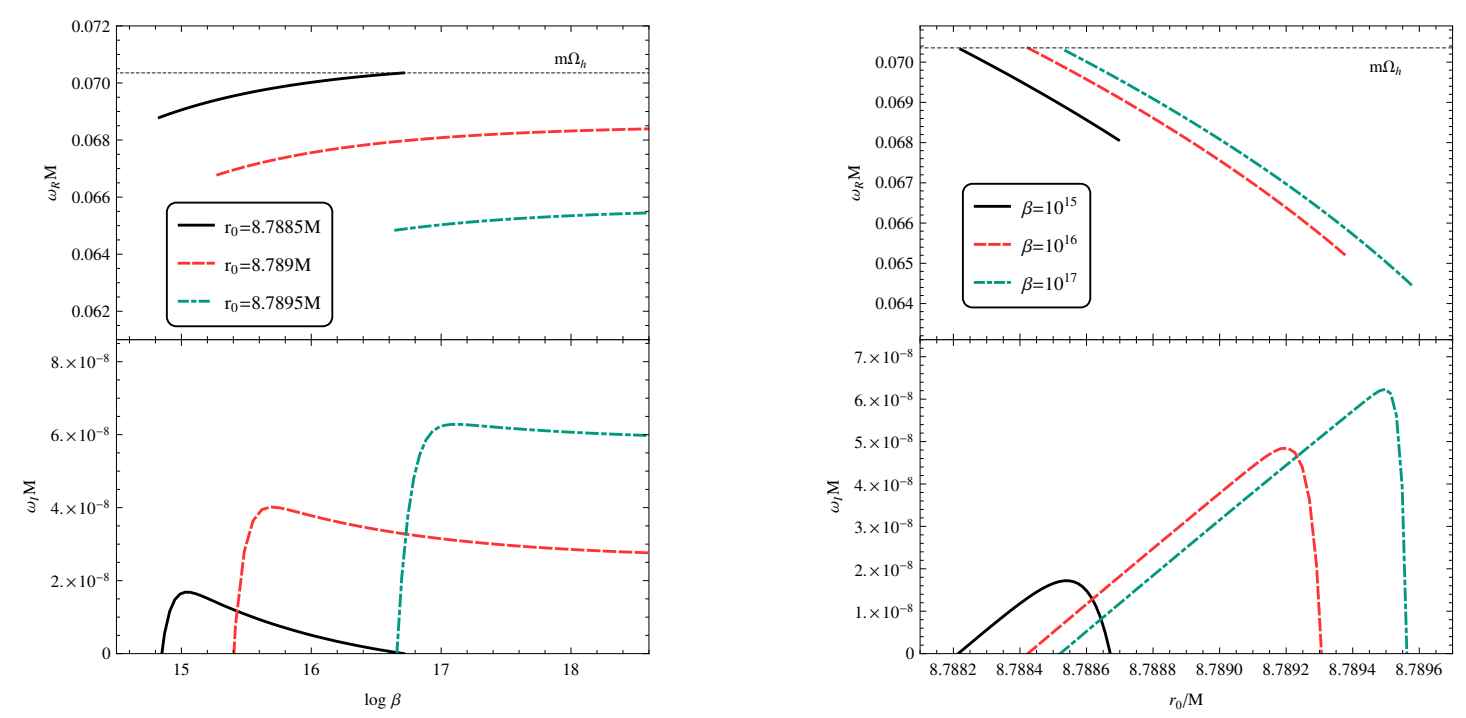

Figure 9. Superradiant instability details for Kerr-de Sitter BH with $a=0.3, \Lambda=0.03$. The BH event horizon and cosmological horizon locate at $r_{h}=2.0431, r_{c}=8.7906$. The angular velocity on the black hole horizon $\Omega_{h}=0.0704$.

the frequency scales with $1 / r_{0}$, we learn that for the Kerr-de Sitter BH with the same cosmological constant but slower rotation, the superradiant instability can occur when the matter shell is put a bit further away with bigger $r_{0}$. Furthermore we observe that with the decrease of $a$, the imaginary part of the frequency falls closer to zero, which indicates that the instability becomes milder. This is understandable because when the BH rotates slower, it will be harder to extract the rotational energy through superradiance. Fixing $\Lambda, r_{0}, \beta$, we show the potential behavior with the change of $a$ in figure 10. It is clearly shown that with the decrease of $a$, the potential barrier to reflect the outgoing wave becomes lower and the potential well becomes shallower. This can be used to account for the observation that lower $a$ makes the superradiant instability milder. We further exhibit the property of the potential when the imaginary part of the frequency reaches the peak for small $\beta$ in figures $4,8,9$ in the left panel of figure 12 . For the limiting $\left(r_{0}, \beta\right)$ to trigger the strongest superradiant instability, we see that for the lower $a$ we have shallower potential barrier to store the reflected perturbation. This again explains the reason that the superradiant instability is weaker when $a$ is smaller.

\subsection{The minimum $a$ to accommodate the superradiant instability}

In the last subsection, we observe that lowering $a$ will make superrandiant instability milder. We can see this from figure 9 where $a=0.3$ and $\Lambda=0.03$. In that case, we need large enough $\beta$ to trigger instability. The physical reason is that it is harder to extract the rotational energy from the Kerr-de Sitter BH background when $a$ is small.

Now the question comes, whether there is the minimum value of $a$ to allow the superradiant instability? We have calculated the result, for $\Lambda=0.1, a_{\min } \sim 0.54$. We calculate the corresponding unstable modes for this critical case, and the results are shown 


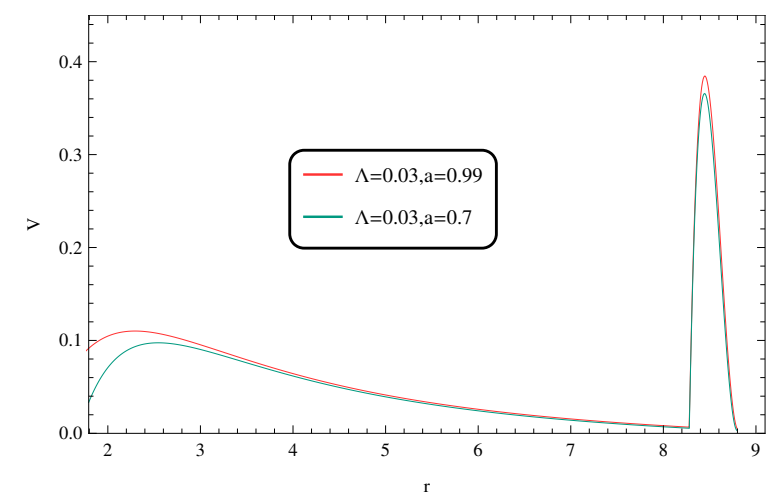

Figure 10. Effective potential behavior with the change of $a$, where we fix $\Lambda=0.03, \beta=10^{7.2}, r_{0}=$ 8.28 .

in figure 12. Comparing to figure 5 with $a=0.99$, we can see that $\beta$ is needed to be very large to spark off superradiant instability and the instability is very mild with very small $\omega_{I}$. Below this $a_{\text {min }}$, no matter how big is the coupling between the matter and the scalar, the superradiant instability will not happen. For the Kerr BH background, we have $a_{\text {min }} \sim 0.18$. We see that $a_{\text {min }}$ increases with the increase of the cosmological constant $\Lambda$. This is reasonable because the increase of $\Lambda$ makes the superradiant instability harder to occur as we discussed above. From the right panel of figure 11, we illustrate the potential behavior of the minimum $a$ for $\Lambda=0.1$ to spark off superradiant instability. We see that at the minimum $a$, the potential is very flattened and the potential well to store the reflected outgoing perturbation is very shallow. Although the potential wall is very high to bounce back the outgoing perturbation, the perturbation will be harder to accumulate near the BH to cause the superradiant instability. This accounts for the very mild instability at this critical case. Below this $a_{\text {min }}$, all the reflected outgoing perturbation will fall inside the BH without any obstacle.

\section{Spontaneous Scalarization}

In $[16,17]$, the authors uncovered a new instability caused by the distribution of matter around BHs. When the matter configuration is dense enough, the $\mathrm{BH}$ is forced to develop scalar hair. This is called the spontaneous scalarization. In their argument, they considered that the matter distribution is spherically symmetric and its backreaction on the geometry is negligible. In this probe limit the background metric is a Schwarzschild BH. They have constructed nonlinear, hairy solutions of ST theories with a Schwarzschild BH at the center.

In this section, we will generalize their argument to the spherically symmetric de Sitter BHs. We focus on the four-dimensional Schwarzschild-de Sitter BH at the center sounded by spherical shell of scalar field. Adopting the decomposition of the scalar field $\varphi(t, r, \theta, \phi)=\sum_{l m} e^{-i \omega t} Y_{l m}(\theta, \phi) \frac{\Psi_{l m}(r)}{r}$, the radial wave equation of the scalar field obeys

$$
\frac{d^{2} \Psi_{l m}(r)}{d r_{*}^{2}}+\left[\omega^{2}-V(r)\right] \Psi_{l m}(r)=0,
$$



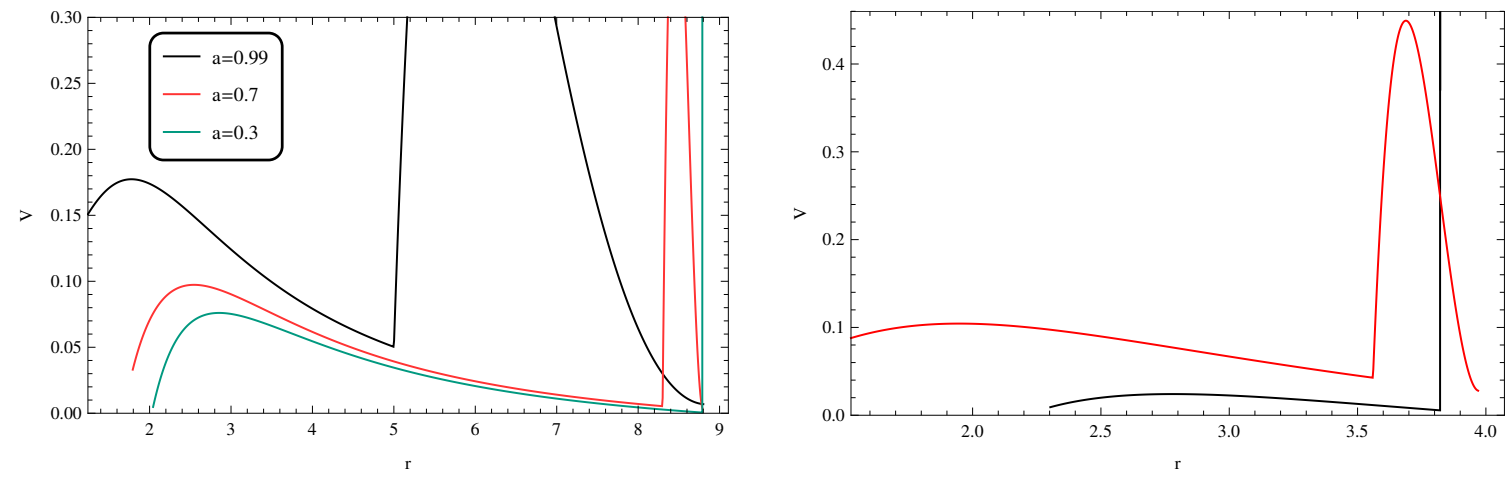

Figure 11. (Left)Effective potential behaviors with the change of $a$. We fix $\Lambda=0.03$ and choose $r_{0}, \beta$ values to have the smallest peak of $\omega_{I}$ in figures 4,8,9. For $a=0.99$, we choose $\left(r_{0}, \beta\right)=\left(5,10^{4.31}\right)$; for $a=0.7$, we select $\left(r_{0}, \beta\right)=\left(8.3,10^{7.325}\right)$; and for $a=0.3$, we adopt $\left(r_{0}, \beta\right)=\left(8.7885,10^{15.03}\right)$. (Right)Effective potential behavior for the minimum $a$ to accommodate superradiant instability when $\Lambda=0.1$ (the black one). For comparison we also plot the line when $a=0.99$ and $\Lambda=0.1$ (the red one). We choose $\left(r_{0}, \beta\right)=\left(3.56,10^{5.46}\right)$ for $a=0.99$ and $\left(r_{0}, \beta\right)=\left(3.82156,10^{14.3879}\right)$ for $a=0.54$, respectively.
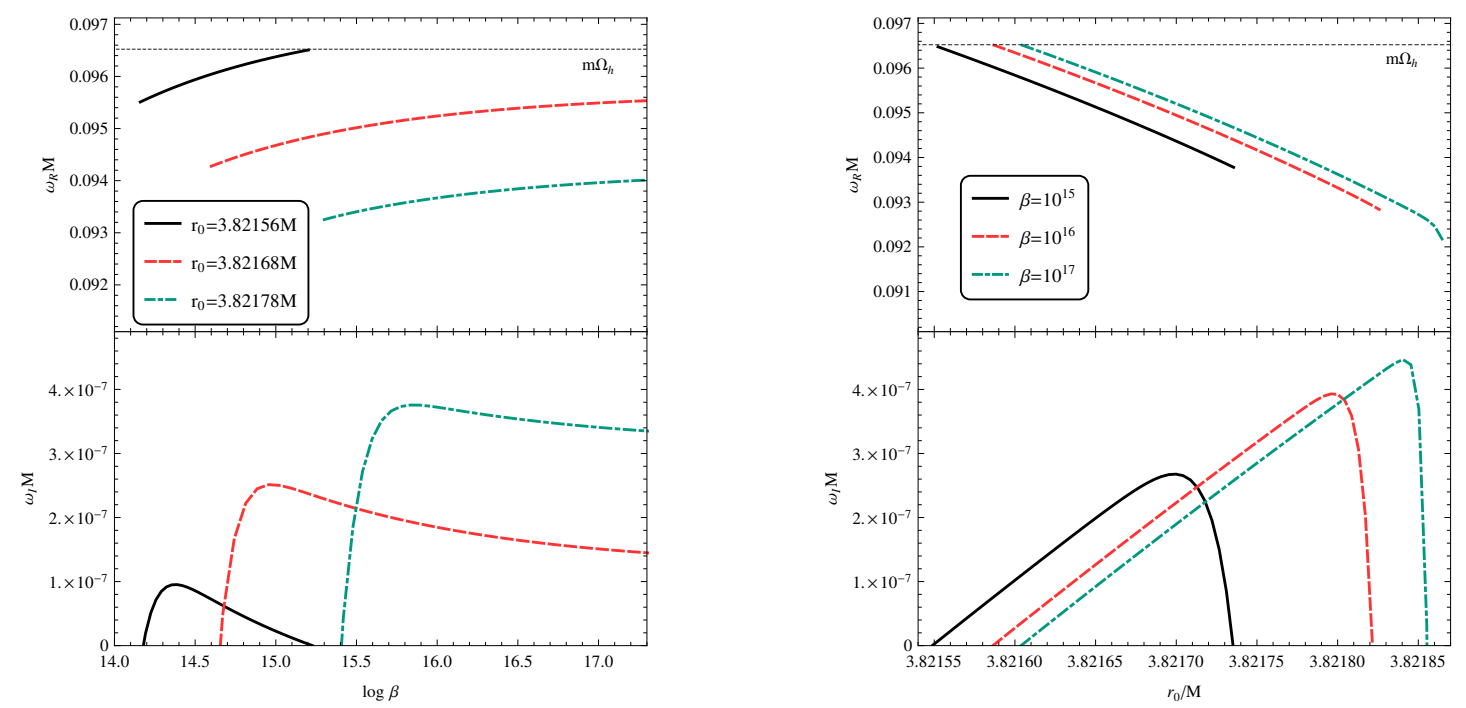

Figure 12. Superradiant instability details for Kerr-de Sitter BH with $a=0.54, \Lambda=0.1$. The BH event horizon and cosmological horizon locate at $r_{h}=2.30281, r_{c}=3.82226$. The angular velocity on the BH horizon $\Omega_{h}=0.0965227$.

in which

$$
\begin{aligned}
V(r) & =f\left(\frac{l(l+1)}{r^{2}}+\frac{1}{r} \frac{d f}{d r}+\mu_{s}^{2}(r)\right), \\
f & =1-\frac{2 M}{r}-\frac{\Lambda}{3} r^{2}
\end{aligned}
$$

Here we have taken the tortoise coordinate defined as $d r / d r_{*}=f . \Psi_{l m}(r)$ is required to satisfy boundary conditions, namely the outgoing wave at the cosmological horizon and 
ingoing wave at the $\mathrm{BH}$ event horizon. The frequency $\omega$ is complex, $\omega=\omega_{R}+i \omega_{I}$. The unstable modes correspond to $\omega_{I}>0$.

According to the well known result in quantum mechanics [55], the sufficient condition for this potential to lead to an instability is $\int_{-\infty}^{\infty} V(r) d r_{*}=\int_{r_{h}}^{r_{c}} \frac{V(r)}{f} d r<0$. Combining with (5.2), this condition becomes

$$
\int_{r_{h}}^{r_{c}} \mu_{s}^{2}(r) d r<-l(l+1)\left(\frac{1}{r_{c}}-\frac{1}{r_{h}}\right)-M\left(\frac{1}{r_{c}^{2}}-\frac{1}{r_{h}^{2}}\right)-\frac{2 \Lambda}{3}\left(r_{c}-r_{h}\right) .
$$

It is obvious that this sufficient condition can be satisfied if the effective mass square is sufficiently negative. It is straightforward to prove that for higher dimensional and charged de Sitter BHs, the instability condition can also be fulfilled if the effective mass square is negative enough.

To understand the instability due to the spontaneous scalarization in the presence of matter, we focus on the final state of spherically symmetric configurations described by

$$
d s^{2}=-h(r) d t^{2}+\frac{1}{f(r)} d r^{2}+r^{2} d \Omega^{2} .
$$

The scalar field $\Phi$ can be integrated from the Klein-Gordon equation, obeying $\partial_{r} \Phi=\frac{Q}{r^{2} \sqrt{f h}}$, where $Q$ is the scalar charge (the integral constant). The BH event horizon $\left(f\left(r_{h}\right)=0\right)$ is surrounded by the matter shell, regularity at the $\mathrm{BH}$ event horizon requires $Q=0$ and $\Phi=\Phi_{-}=$const inside the matter shell. For the same reason, at the cosmological horizon out of the matter shell, $f\left(r_{c}\right)=0$, which also demands $\Phi=\Phi_{+}=$const there. So the scalar field can only be constants both inside and outside the matter shell. However, these two constants, $\Phi_{-}$and $\Phi_{+}$, can be different due to the existence of the matter shell. That is, there is a finite jump of the scalar field over the matter shell, which leads to a nontrivial configuration. This configuration is very different from that discussed in $[16,17]$ without cosmological horizon but only $\mathrm{BH}$ event horizon in the final spherical configuration, which does not have regularity condition out of the matter shell so that the scalar field $\Phi$ is not limited to be a constant out of the shell and can develop a coordinate-dependent profile $[16,17]$.

In our case, due to the constant profile of the scalar field inside and outside the matter shell, the final metric on both sides will take Schwarzschild-de Sitter forms but with different parameters $(M, \Lambda)$. The cosmological constants $\Lambda_{ \pm}$on both sides are related to the scalar field by the relation $\Lambda_{ \pm}=V\left(\Phi_{ \pm}\right)$. If the matter shell is made of perfect fluid, the surface stress-energy tensor takes the form

$$
S_{a b}^{E}=\sigma u_{a} u_{b}+P\left(\gamma_{a b}+u_{a} u_{b}\right),
$$

where $\sigma, P$ denote density and pressure respectively. $u_{a}$ is the on-shell four velocity and $\gamma_{a b}$ is the induced metric on the shell. According to the Israel-Darmois junction condition, the jump of the metric over the matter shell can be expressed in terms of the shell composition. 
In particular, for a static shell at $r=R$, we have $[16,17,57]$

$$
\begin{aligned}
\sigma & =-\frac{1}{4 \pi R}\left(\sqrt{f_{+}}-\sqrt{f_{-}}\right) \\
P & =\frac{1}{8 \pi R}\left(-4 \pi R \sigma+\sqrt{f_{+}} \frac{R f_{+}^{\prime}}{2 f_{+}}-\sqrt{f_{-}} \frac{R f_{-}^{\prime}}{2 f_{-}}\right) .
\end{aligned}
$$

In summary, in spherical de Sitter BH background, spontaneous scalarization can also happen but in a special style. We should note that the jump of the scalar field over the matter shell discussed above is due to the simplification of the thin shell. In a more realistic configuration, matter will have non-vanishing support as treated in [7-11].

\section{Summary and discussion}

In this paper, we have studied the stability of Kerr-de Sitter BH surrounded by matter shell in ST theories. We have found that similar to the asymptotically flat Kerr BH [16, 17], there exists superradiant instability in the Kerr-de Sitter BH configurations.

In the Kerr-de Sitter BH background, we have observed that with the increase of the cosmological constant, the superradiant instability becomes harder to be triggered. It needs to put the matter shell closer to the hole and increase the coupling between the matter and scalar field to spark off the instability. We have got the physical understanding of the phenomenon we observed by examining the potential behavior.

Further, we have examined the influence of the angular momentum per unit mass on the superradiant instability. We have disclosed that slower rotation will make the superradiant instability harder to happen. There is a minimum value of the angular momentum per unit mass to allow the appearance of the superradiant instability. This minimum $a$ increases when the cosmological constant becomes bigger. The physical reasons behind this phenomenon have also been explained.

On the superradiant instability study, the difference between asymptotically flat Kerr black hole and Kerr-de Sitter BH is very little. The main reason is that the superradiant instability is mainly influenced by the potential barrier to reflect back the outgoing perturbation and the potential well between the $\mathrm{BH}$ horizon and the potential barrier to store the reflected perturbation. It has little to do with the boundary condition at the infinity for the asymptotically flat spacetime or at the cosmological horizon for the de Sitter spacetime.

We have also examined the spontaneous scalarization in the de Sitter BH background. Different from the superradiant instability, now the boundary at the cosmological horizon becomes important in the investigation. Different from the asymptotically flat BH case [16, 17], the regularity condition at the cosmological horizon enforces the scalarization to take a special style, that is the scalar field takes a constant profile outside the shell as well as inside the shell in the spherical de Sitter BH background. Since we only focused on the spherical de Sitter configurations, one question we may ask is that whether a more general scalarization can happen once the de Sitter BH background starts to rotate. Further investigations on this topic are called for. 


\section{Acknowledgments}

We thank De-Chang Dai, Xiao-Mei Kuang, De-Cheng Zou, Zhi-Ying Zhu and Yun-Qi Liu for their helpful suggestions and discussions. This work was supported in part by the National Natural Science Foundation of China.

Open Access. This article is distributed under the terms of the Creative Commons Attribution License (CC-BY 4.0), which permits any use, distribution and reproduction in any medium, provided the original author(s) and source are credited.

\section{References}

[1] C. Brans and R.H. Dicke, Mach's principle and a relativistic theory of gravitation, Phys. Rev. 124 (1961) 925 [INSPIRE].

[2] Y. Fujii and K. Maeda, The scalar-tensor theory of gravitation, University Press, Cambridge, U.S.A. (2003).

[3] V. Faraoni, Cosmology in scalar tensor gravity, Springer (2004) [ISBN-1-4020-1988-2].

[4] M.B. Green, J.H. Schwarz and E. Witten, Superstring Theory. Vol. 2: Loop Amplitudes, Anomalies And Phenomenology, University Press, Cambridge, U.K. (1987).

[5] J. Khoury and A. Weltman, Chameleon fields: Awaiting surprises for tests of gravity in space, Phys. Rev. Lett. 93 (2004) 171104 [astro-ph/0309300] [InSPIRE].

[6] K. Hinterbichler and J. Khoury, Symmetron Fields: Screening Long-Range Forces Through Local Symmetry Restoration, Phys. Rev. Lett. 104 (2010) 231301 [arXiv:1001.4525] [INSPIRE].

[7] T. Damour and G. Esposito-Farese, Nonperturbative strong field effects in tensor - scalar theories of gravitation, Phys. Rev. Lett. 70 (1993) 2220 [INSPIRE].

[8] T. Damour and G. Esposito-Farese, Tensor - scalar gravity and binary pulsar experiments, Phys. Rev. D 54 (1996) 1474 [gr-qc/9602056] [InSPIRE].

[9] P. Pani, V. Cardoso, E. Berti, J. Read and M. Salgado, The vacuum revealed: the final state of vacuum instabilities in compact stars, Phys. Rev. D 83 (2011) 081501 [arXiv:1012.1343] [INSPIRE].

[10] E. Barausse, C. Palenzuela, M. Ponce and L. Lehner, Neutron-star mergers in scalar-tensor theories of gravity, Phys. Rev. D 87 (2013) 081506 [arXiv: 1212.5053] [InSPIRE].

[11] C. Palenzuela, E. Barausse, M. Ponce and L. Lehner, Dynamical scalarization of neutron stars in scalar-tensor gravity theories, Phys. Rev. D 89 (2014) 044024 [arXiv:1310.4481] [INSPIRE].

[12] S.W. Hawking, Black holes in the Brans-Dicke theory of gravitation, Commun. Math. Phys. 25 (1972) 167 [INSPIRE].

[13] T.P. Sotiriou and V. Faraoni, Black holes in scalar-tensor gravity, Phys. Rev. Lett. 108 (2012) 081103 [arXiv:1109.6324] [InSPIRE].

[14] V. Cardoso, S. Chakrabarti, P. Pani, E. Berti and L. Gualtieri, Floating and sinking: The Imprint of massive scalars around rotating black holes, Phys. Rev. Lett. 107 (2011) 241101 [arXiv: 1109.6021] [INSPIRE]. 
[15] N. Yunes, P. Pani and V. Cardoso, Gravitational Waves from Quasicircular Extreme Mass-Ratio Inspirals as Probes of Scalar-Tensor Theories, Phys. Rev. D 85 (2012) 102003 [arXiv:1112.3351] [INSPIRE].

[16] V. Cardoso, I.P. Carucci, P. Pani and T.P. Sotiriou, Matter around Kerr black holes in scalar-tensor theories: scalarization and superradiant instability, Phys. Rev. D 88 (2013) 044056 [arXiv: 1305.6936] [INSPIRE].

[17] V. Cardoso, I.P. Carucci, P. Pani and T.P. Sotiriou, Black holes with surrounding matter in scalar-tensor theories, Phys. Rev. Lett. 111 (2013) 111101 [arXiv:1308.6587] [INSPIRE].

[18] S. Weinberg, Cosmology, Oxford University Press, Oxford, U.K. (2008).

[19] S. Hod, Stability of the extremal Reissner-Nordström black hole to charged scalar perturbations, Phys. Lett. B 713 (2012) 505 [arXiv: 1304.6474] [INSPIRE].

[20] V. Cardoso, Black hole bombs and explosions: from astrophysics to particle physics, Gen. Rel. Grav. 45 (2013) 2079 [arXiv:1307.0038] [INSPIRE].

[21] S. Yoshida, N. Uchikata and T. Futamase, Quasinormal modes of Kerr-de Sitter black holes, Phys. Rev. D 81 (2010) 044005 [InSPIRE].

[22] T.P. Sotiriou, V. Faraoni and S. Liberati, Theory of gravitation theories: A No-progress report, Int. J. Mod. Phys. D 17 (2008) 399 [arXiv:0707.2748] [INSPIRE].

[23] T. Damour and G. Esposito-Farese, Gravitational wave versus binary - pulsar tests of strong field gravity, Phys. Rev. D 58 (1998) 042001 [gr-qc/9803031] [INSPIRE].

[24] P.C.C. Freire, N. Wex, G. Esposito-Farese, J.P.W. Verbiest, M. Bailes et al., The relativistic pulsar-white dwarf binary PSR J1738+0333 II. The most stringent test of scalar-tensor gravity, Mon. Not. Roy. Astron. Soc. 423 (2012) 3328 [arXiv:1205.1450] [InSPIRE].

[25] S.A. Teukolsky, Perturbations of a rotating black hole. 1. Fundamental equations for gravitational electromagnetic and neutrino field perturbations, Astrophys. J. 185 (1973) 635 [INSPIRE].

[26] E.D. Fackerell, R.G. Crossman, Spin-weighted angular spheroidal functions, J. Math. Phys. 18 (1977) 1849.

[27] E. Seidel, A Comment on the Eigenvalues of Spin Weighted Spheroidal Functions, Class. Quant. Grav. 6 (1989) 1057 [INSPIRE].

[28] E.W. Leaver, An Analytic Representation for the Quasi-Normal Modes of Kerr Black Holes, Proc. Roy. Soc. Lond. A 402 (1985) 285.

[29] E.W. Leaver, Spectral decomposition of the perturbation response of the Schwarzschild geometry, Phys. Rev. D 34 (1986) 384 [INSPIRE].

[30] E.W. Leaver, Solutions to a generalized spheroidal wave equation: Teukolsky's equations in general relativity, and the two-center problem in molecular quantum mechanics, J. Math. Phys. 27 (1986) 1238.

[31] H. Suzuki, E. Takasugi and H. Umetsu, Analytic solutions of Teukolsky equation in Kerr-de Sitter and Kerr-Newman-de Sitter geometries, Prog. Theor. Phys. 102 (1999) 253 [gr-qc/9905040] [INSPIRE].

[32] E. Berti, V. Cardoso and M. Casals, Eigenvalues and eigenfunctions of spin-weighted spheroidal harmonics in four and higher dimensions, Phys. Rev. D 73 (2006) 024013 [Erratum ibid. D 73 (2006) 109902] [gr-qc/0511111] [rNSPIRE]. 
[33] H. Suzuki, E. Takasugi and H. Umetsu, Perturbations of Kerr-de Sitter black hole and Heun's equations, Prog. Theor. Phys. 100 (1998) 491 [gr-qc/9805064] [InSPIRE].

[34] H.T. Cho, A.S. Cornell, J. Doukas and W. Naylor, Asymptotic iteration method for spheroidal harmonics of higher-dimensional Kerr-(A)dS black holes, Phys. Rev. D 80 (2009) 064022 [arXiv: 0904.1867] [INSPIRE].

[35] P.R. Brady, C.M. Chambers, W.G. Laarakkers and E. Poisson, Radiative falloff in Schwarzschild-de Sitter space-time, Phys. Rev. D 60 (1999) 064003 [gr-qc/9902010] [INSPIRE].

[36] C. Molina, D. Giugno, E. Abdalla and A. Saa, Field propagation in de Sitter black holes, Phys. Rev. D 69 (2004) 104013 [gr-qc/0309079] [inSPIRE].

[37] E. Berti, V. Cardoso and A.O. Starinets, Quasinormal modes of black holes and black branes, Class. Quant. Grav. 26 (2009) 163001 [arXiv:0905.2975] [INSPIRE].

[38] R.A. Konoplya and A. Zhidenko, Quasinormal modes of black holes: From astrophysics to string theory, Rev. Mod. Phys. 83 (2011) 793 [arXiv:1102.4014] [INSPIRE].

[39] G. Pöschl and E. Teller, Bemerkungen zur Quantenmechanik des anharmonischen Oszillators, Z. Phys. 83 (1933) 143 [INSPIRE].

[40] V. Cardoso and J.P.S. Lemos, Quasinormal modes of the near extremal Schwarzschild-de Sitter black hole, Phys. Rev. D 67 (2003) 084020 [gr-qc/0301078] [INSPIRE].

[41] V. Cardoso, M. Lemos and M. Marques, On the instability of Reissner-Nordstrom black holes in de Sitter backgrounds, Phys. Rev. D 80 (2009) 127502 [arXiv:1001.0019] [InSPIRE].

[42] C. Gundlach, R.H. Price and J. Pullin, Late time behavior of stellar collapse and explosions: 1. Linearized perturbations, Phys. Rev. D 49 (1994) 883 [gr-qc/9307009] [INSPIRE].

[43] C. Gundlach, R.H. Price and J. Pullin, Late time behavior of stellar collapse and explosions: 2. Nonlinear evolution, Phys. Rev. D 49 (1994) 890 [gr-qc/9307010] [INSPIRE].

[44] R.A. Konoplya and A. Zhidenko, Instability of higher dimensional charged black holes in the de-Sitter world, Phys. Rev. Lett. 103 (2009) 161101 [arXiv:0809.2822] [INSPIRE].

[45] G. Wentzel, Eine Verallgemeinerung der Quantenbedingungen für die Zwecke der Wellenmechanik, Z. Physik 38 (1926) 518.

[46] K.A. Kramers Wellenmechanik und halbzahlige Quantisierung, Z. Physik 39 (1926) 828.

[47] L. Brillouin, La mécanique ondulatoire de Schrödinger: une méthode générale de resolution par approximations successives, Compt. Rend. 183 (1926) 24.

[48] S. Chandrasekhar and S. Detweiler, The Quasi-Normal Modes of the Schwarzschild Black Hole, Proc. R. Soc. Lond. A 344 (1975) 441.

[49] C. Molina, P. Pani, V. Cardoso and L. Gualtieri, Gravitational signature of Schwarzschild black holes in dynamical Chern-Simons gravity, Phys. Rev. D 81 (2010) 124021 [arXiv: 1004 .4007] [INSPIRE].

[50] U. Khanal, Further investigations of the Kerr-de Sitter space, Phys. Rev. D 32 (1985) 879 [INSPIRE].

[51] T. Tachizawa and K.-ichi Maeda, Superradiance in the Kerr-de Sitter space-time, Phys. Lett. A 172 (1993) 325. 
[52] I.G. Moss and J.P. Norman, Gravitational quasinormal modes for anti-de Sitter black holes, Class. Quant. Grav. 19 (2002) 2323 [gr-qc/0201016] [inSPIRE].

[53] S. Yoshida and T. Futamase, Numerical analysis of quasinormal modes in nearly extremal Schwarzschild-de Sitter space-times, Phys. Rev. D 69 (2004) 064025 [gr-qc/0308077] [INSPIRE].

[54] A. Maassen van den Brink, Approach to the extremal limit of the Schwarzschild-de Sitter black hole, Phys. Rev. D 68 (2003) 047501 [gr-qc/0304092] [InSPIRE].

[55] W.F. Buell and B.A. Shadwick, Potentials and bound states, Am. J. Phys. 63 (1995) 256.

[56] V. Cardoso and S. Yoshida, Superradiant instabilities of rotating black branes and strings, JHEP 07 (2005) 009 [hep-th/0502206] [INSPIRE].

[57] W. Israel, Singular hypersurfaces and thin shells in general relativity, Nuovo Cim. B 44S10 (1966) 1 [Erratum ibid. B 48 (1967) 463] [INSPIRE]. 